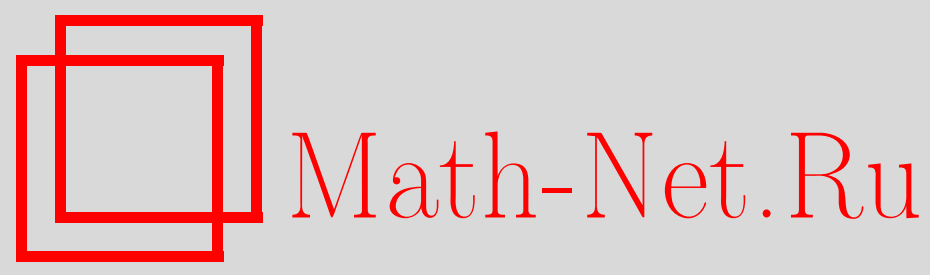

Нгуен Минь Чи, Об уравнении Грушина, Матем. заметки, 1998, том 63, выпуск 1, 95-105

DOI: https://doi.org/10.4213/mzm1251

Использование Общероссийского математического портала Math-Net.Ru подразумевает, что вы прочитали и согласны с пользовательским соглашением http://www . mathnet.ru/rus/agreement

Параметры загрузки:

IP : 52.205.19.152

26 апреля 2023 г., $17: 14: 03$

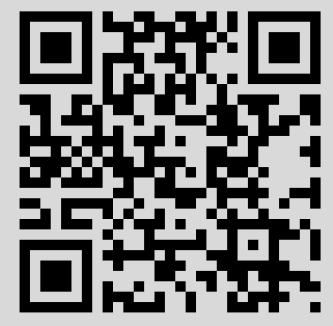


УДК 517

\title{
ОБ УРАВНЕНИИ ГРУШИНА
}

\section{Нгуен Минь Чи}

\begin{abstract}
В статье рассмотрена нелинейная задача для уравнения типа Грушина. Используя тождество Похожаева, докажем для таких уравнений некоторые резултаты об отсутствии нетривиальных решений. Остальная часть связана с гладкостью собственных функций вблизи границы. Для получения некоторых результатов используется явное выражение фундаментального решения и преобразования Кельвина
\end{abstract}

Библиография: 9 названий.

1. Введение. В этой статье мы рассматриваем вырождающиеся эллиптические уравнения

$$
L=\frac{\partial^{2}}{\partial x_{1}^{2}}+\varphi^{2}\left(x_{1}\right) \frac{\partial^{2}}{\partial x_{2}^{2}}
$$

в $\mathbb{R}^{2}$. Если $\varphi^{2}\left(x_{1}\right)=x_{1}^{2 k}, k$-целое число, два векторных поля

$$
X_{1}=\frac{\partial}{\partial x_{1}}, \quad X_{2}=x_{1}^{k} \frac{\partial}{\partial x_{2}}
$$

удовлетворяют условию Хёрмандера, то $L$ является гипоэллиптическим оператором. В предельном случае вырожденности: $\varphi\left(x_{1}\right) \in C^{\infty}\left(\mathbb{R}^{1}\right), \varphi\left(x_{1}\right) \neq 0$, если $x_{1} \neq 0, \varphi\left(x_{1}\right)$ принимает действительные значения,

$$
\frac{d^{n} \varphi(0)}{d x_{1}^{n}}=0
$$

для любого $n$; оператор $L$ также является гипоэллиптическим оператором (хотя условие Хёрмандера не выполнено, см. [1]). Подробное исследование гладкости решений этих уравнений вблизи границы было изложено в [2]. В [3] было замечено, что значение $(\alpha+2) / \alpha$ ведет себя как критический показатель Соболева для оператора

$$
G_{\alpha}=\frac{\partial^{2}}{\partial x_{1}^{2}}+\left|x_{1}\right|^{2 \alpha} \frac{\partial^{2}}{\partial x_{2}^{2}}, \quad \alpha>0 .
$$

Аналогичные результаты были получены в [4] в связи с изучением нелинейных задач для $\square_{b}$ на группе Гейзенберга. Коротко напомним главные результаты из [3].

Теорема 1. Пусть $\Omega$ является $G_{\alpha}$-звездной по отношению $\kappa$ точке $\left(x_{1}, x_{2}\right)=$ $(0,0)$. Тогда следующая граничная задача:

$$
\begin{gathered}
G_{\alpha} u+\lambda u+|u|^{\gamma} u=0 \quad \text { в } \Omega, \\
u=0 \quad \text { на } \partial \Omega,
\end{gathered}
$$

где $\lambda \leqslant 0, \gamma>4 / \alpha$, не имеет нетривиальных решений в $H^{2}(\Omega)$. 
ОПРЕДЕЛЕниЕ 1 . Через $S_{\alpha}^{1, p}(\Omega), 1 \leqslant p<\infty$, мы обозначим множество всех функций $u \in L^{p}(\Omega)$ таких, что

$$
\frac{\partial u}{\partial x_{1}} \in L^{p}(\Omega),\left|x_{1}\right|^{\alpha} \frac{\partial u}{\partial x_{2}} \in L^{p}(\Omega) .
$$

Мы определим норму в этом пространстве следуюшим образом:

$$
\|u\|_{S_{\alpha}^{1, p}(\Omega)}=\left\{\int_{\Omega}\left(|u|^{p}+\left|\frac{\partial u}{\partial x_{1}}\right|^{p}+\left.\left.|| x_{1}\right|^{\alpha} \frac{\partial u}{\partial x_{2}}\right|^{p}\right) d x_{1} d x_{2}\right\}^{1 / p} .
$$

Если $p=2$, мы можем определить скалярное произведение в $S_{\alpha}^{1,2}(\Omega)$ как

$$
(u, v)_{S_{\alpha}^{1,2}(\Omega)}=(u, v)_{L^{2}(\Omega)}+\left(\frac{\partial u}{\partial x_{1}}, \frac{\partial v}{\partial x_{1}}\right)_{L^{2}(\Omega)}+\left(\left|x_{1}\right|^{\alpha} \frac{\partial u}{\partial x_{2}},\left|x_{1}\right|^{\alpha} \frac{\partial v}{\partial x_{2}}\right)_{L^{2}(\Omega)} .
$$

Легко показать, что $S_{\alpha}^{1, p}(\Omega)$ является банаховым пространством и $S_{\alpha}^{1,2}(\Omega)$ - гильбертовым пространством. Пространство $S_{\alpha, 0}^{1, p}(\Omega)$ определяется как замыкание $C_{0}^{1}(\Omega)$ в пространстве $S_{\alpha}^{1, p}(\Omega)$.

ТЕОрема 2. 1) Ecлu $1 \leqslant p<\alpha+2$, mo $S_{\alpha, 0}^{1, p}(\Omega) \subset L^{((\alpha+2) p) /(\alpha+2-p)-\tau}(\Omega)$ для $\Omega ю-$ бого положительного достаточно малого $\tau, u$ это вложсение вполне непрерывно.

2) $\operatorname{Ecли~} p>\alpha+2$, mo $S_{\alpha, 0}^{p}(\Omega) \subset C^{0}(\bar{\Omega})$.

Теорема 3. Предположим, что $f(u)$ удовлетворяет следующим условиям:

1) $f \in C_{\mathrm{loc}}^{0, \beta}(\mathbb{R}), f(0)=0$;

2) $|f(u)| \leqslant C\left(1+|u|^{m}\right) c 1<m<(\alpha+4) / k$;

3) $f(u)=o(u)$, когда $u \rightarrow 0$;

4) существует число $A$ такое, что $|u| \geqslant A$,

$$
\int_{0}^{u} f(s) d s \leqslant \mu f(u) u
$$

əде $\mu \in[0,1 / 2)$.

Тогда граничная задача

$$
\begin{gathered}
G_{\alpha} u+f(u)=0 \quad \text { в } \Omega, \\
u=0 \quad \text { на } \partial \Omega,
\end{gathered}
$$

всегда имеет нетривиальное решение в $S_{\alpha, 0}^{1, p}(\Omega)$.

2. Нелинейные уравнения для оператора Грушина. Заметим, что $(\alpha+2) / \alpha \rightarrow$ $\infty$ при $\alpha \rightarrow 0$. Этот факт характеризует известную ситуацию: предельньй случай (оператор Лапласа в $\mathbb{R}^{2}$ ) не имеет критического показателя (см. [5]). Когда $\alpha \rightarrow \infty$, имеем $(\alpha+2) / \alpha \rightarrow 1$. Приведем некоторые результаты, которые характеризуют сказанное выше.

Рассмотрим задачу

$$
\begin{gathered}
L u+f(u)=\frac{\partial^{2} u}{\partial x_{1}^{2}}+\varphi^{2}\left(x_{1}\right) \frac{\partial^{2} u}{\partial x_{2}^{2}}+f(u)=0 \text { в } \Omega, \\
u=0 \text { на } \partial \Omega,
\end{gathered}
$$

где $f(0)=0, f(u) \in C(\mathbb{R})$ и $\Omega$ - ограниченная область, содержащая начало координат, с гладкой границей в $\mathbb{R}^{2}$. Положим

$$
F(u)=\int_{0}^{u} f(s) d s
$$

$\nu=\left(\nu_{1}, \nu_{2}\right)$ - внешняя нормаль к $\partial \Omega$. Через $C$ обозначим обшие константы, которые не зависят от функций, но могут менять их значения. 
ОПРЕДЕЛЕНИЕ 2. Область $\Omega$ называется $L_{\varphi, \beta}$-звездной по отношению $\kappa$ точ$\kappa e(0,0)$, если неравенство $\left(\nu_{1}^{2}+\varphi^{2}\left(x_{1}\right) \cdot \nu_{2}^{2}\right)\left(x_{1} \cdot \nu_{1}+(\beta+1) x_{2} \cdot \nu_{2}\right)>0$ выполняется почти всюду на $\partial \Omega$.

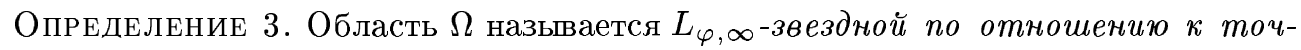
$\kappa e(0,0)$, если $\Omega$ является $L_{\varphi, \beta}$-звездной для любого $\beta>0$.

ПримеР 1. Единичный шар $B_{r}=\left\{\left(x_{1}, x_{2}\right) \mid x_{1}^{2}+x_{2}^{2}<r^{2}\right\}$ представляет собой $L_{\varphi, \infty}$-звездную область.

ЛЕмма (тождество Похожаева). Пусть $и(x)$ - решение задачи (1), (2), которое принадлежит $H^{2}(\Omega)$. Тогда $и(x)$ удовлетворяет тохсдеству

$$
\begin{aligned}
\int_{\Omega}\left\{(\beta+1) F(u)-\frac{\beta-1}{2} f(u) u\right\} d x_{1} d x_{2} \\
=\frac{1}{2} \int_{\partial \Omega}\left(\frac{\partial u}{\partial \nu}\right)^{2}\left(\nu_{1}^{2}+\varphi^{2}\left(x_{1}\right) \cdot \nu_{2}^{2}\right)\left\{x_{1} \cdot \nu_{1}+\beta x_{2} \cdot \nu_{2}\right\} d s \\
\quad+\int_{\Omega}\left(x_{1} \varphi^{\prime}\left(x_{1}\right) \varphi\left(x_{1}\right)-\beta \varphi^{2}\left(x_{1}\right)\right)\left(\frac{\partial u}{\partial x_{2}}\right)^{2} d x_{1} d x_{2}
\end{aligned}
$$

для любого $\beta>0$.

ДокАЗАТЕЛьСтво. По теореме вложения Соболева для ограниченных областей с гладкой границей имеем $H^{2}(\Omega) \subset C^{0, \alpha}(\bar{\Omega})$, где $0<\alpha<1$. Заметим, что

$$
\frac{\partial}{\partial x_{1}}\left(x_{1} F(u)\right)=F(u)+x_{1} f(u) \frac{\partial u}{\partial x_{1}}, \quad \frac{\partial}{\partial x_{2}}\left(x_{2} F(u)\right)=F(u)+x_{2} f(u) \frac{\partial u}{\partial x_{2}} .
$$

Из формулы Гаусса-Остроградского вьводим

$$
\int_{\Omega} F(u) d x_{1} d x_{2}=-\int_{\Omega} x_{1} f(u) \frac{\partial u}{\partial x_{1}} d x_{1} d x_{2}
$$

откуда

$$
\beta \int_{\Omega} F(u) d x_{1} d x_{2}=-\beta \int_{\Omega} x_{2} f(u) \frac{\partial u}{\partial x_{2}} d x_{1} d x_{2}
$$

Следовательно,

$$
\begin{aligned}
(1+\beta) \int_{\Omega} F(u) d x_{1} d x_{2} & =-\int_{\Omega}\left(x_{1} \frac{\partial u}{\partial x_{1}}+\beta x_{2} \frac{\partial u}{\partial x_{2}}\right) f(u) d x_{1} d x_{2} \\
& =\int_{\Omega}\left(x_{1} \frac{\partial u}{\partial x_{1}}+\beta x_{2} \frac{\partial u}{\partial x_{2}}\right)\left(\frac{\partial^{2} u}{\partial x_{1}^{2}}+\varphi^{2}\left(x_{1}\right) \frac{\partial^{2} u}{\partial x_{2}^{2}}\right) d x_{1} d x_{2}
\end{aligned}
$$


Снова применяя формулу Гаусса-Остроградского, получаем (подробное вычисление можно найти в [6])

$$
\begin{aligned}
(1+\beta) & \int_{\Omega} F(u) d x_{1} d x_{2}=-\int_{\Omega}\left(x_{1} \frac{\partial u}{\partial x_{1}}+\beta x_{2} \frac{\partial u}{\partial x_{2}}\right) f(u) d x_{1} d x_{2} \\
= & -\frac{1}{2} \int_{\Omega}\left(\frac{\partial u}{\partial x_{1}}\right)^{2} d x_{1} d x_{2}+\frac{1}{2} \int_{\Omega}\left(2 x_{1} \varphi^{\prime}\left(x_{1}\right) \varphi\left(x_{1}\right)+\varphi^{2}\left(x_{1}\right)\right)\left(\frac{\partial u}{\partial x_{2}}\right)^{2} d x_{1} d x_{2} \\
& +\frac{1}{2} \int_{\partial \Omega}\left(x_{1} \cdot \nu_{1}\right)\left(\frac{\partial u}{\partial x_{1}}\right)^{2} d s-\frac{1}{2} \int_{\partial \Omega}\left(x_{1} \cdot \varphi^{2}\left(x_{1}\right) \cdot \nu_{1}\right)\left(\frac{\partial u}{\partial x_{2}}\right)^{2} d s \\
& -\int_{\partial \Omega}\left(x_{1} \cdot \varphi^{2}\left(x_{1}\right) \cdot \nu_{2}\right) \frac{\partial u}{\partial x_{1}} \frac{\partial u}{\partial x_{2}} d s-\frac{\beta}{2} \int_{\Omega} \varphi^{2}\left(x_{1}\right) \cdot\left(\frac{\partial u}{\partial x_{2}}\right)^{2} d x_{1} d x_{2} \\
& +\frac{\beta}{2} \int_{\partial \Omega}\left(x_{2} \cdot \varphi^{2}\left(x_{1}\right) \cdot \nu_{2}\right)\left(\frac{\partial u}{\partial x_{2}}\right)^{2} d s+\frac{\beta}{2} \int_{\Omega}\left(\frac{\partial u}{\partial x_{1}}\right)^{2} d x_{1} d x_{2} \\
& -\frac{\beta}{2} \int_{\partial \Omega}\left(x_{2} \cdot \nu_{2}\right)\left(\frac{\partial u}{\partial x_{1}}\right)^{2} d s+\beta \int_{\partial \Omega}\left(x_{2} \cdot \nu_{1}\right) \frac{\partial u}{\partial x_{2}} \frac{\partial u}{\partial x_{1}} d s .
\end{aligned}
$$

Так как

$$
\frac{\partial}{\partial x_{1}}=\nu_{1} \cdot \frac{\partial}{\partial \nu}, \quad \frac{\partial}{\partial x_{2}}=\nu_{2} \cdot \frac{\partial}{\partial \nu},
$$

поэтому имеем

$$
\begin{aligned}
(1+ & \beta) \int_{\Omega} F(u) d x_{1} d x_{2}=\frac{\beta-1}{2} \int_{\Omega}\left(\frac{\partial u}{\partial x_{1}}\right)^{2} d x_{1} d x_{2} \\
& +\int_{\Omega}\left\{x_{1} \varphi^{\prime}\left(x_{1}\right) \varphi\left(x_{1}\right)+\left(\frac{1-\beta}{2}\right) \varphi^{2}\left(x_{1}\right)\right\}\left(\frac{\partial u}{\partial x_{2}}\right)^{2} d x_{1} d x_{2} \\
& +\frac{1}{2} \int_{\partial \Omega}\left(\frac{\partial u}{\partial \nu}\right)^{2}\left(\nu_{1}^{2}+\varphi^{2}\left(x_{1}\right) \cdot \nu_{2}^{2}\right)\left\{x_{1} \cdot \nu_{1}+\beta x_{2} \cdot \nu_{2}\right\} d s
\end{aligned}
$$

Значит,

$$
\begin{aligned}
\int_{\Omega}\left\{(\beta+1) F(u)-\frac{\beta-1}{2} f(u) u\right\} d x_{1} d x_{2} \\
=\frac{1}{2} \int_{\partial \Omega}\left(\frac{\partial u}{\partial \nu}\right)^{2}\left(\nu_{1}^{2}+\varphi^{2}\left(x_{1}\right) \cdot \nu_{2}^{2}\right)\left\{x_{1} \cdot \nu_{1}+\beta x_{2} \cdot \nu_{2}\right\} d s \\
\quad+\int_{\Omega}\left(x_{1} \varphi^{\prime}\left(x_{1}\right) \varphi\left(x_{1}\right)-\beta \varphi^{2}\left(x_{1}\right)\right)\left(\frac{\partial u}{\partial x_{2}}\right)^{2} d x_{1} d x_{2} .
\end{aligned}
$$

Следующие две теоремы очевидным образом следуют из доказанной леммы.

TЕОРема 4. Пусть $\Omega-L_{\beta}$-звездная по отношению $к$ точке $(0,0) u$

1) $(\beta+2) F(u)-\frac{\beta}{2} f(u)<0$, когда $u \neq 0$;

2) $x_{1} \cdot \varphi^{\prime}\left(x_{1}\right) \geqslant(\beta+1) \varphi\left(x_{1}\right)$ в $\Omega$.

Тогда не существует нетривиальное решение $и \in H^{2}(\Omega)$ для задачи (1), (2). 
ТЕорема 5. Пусть $\Omega-L_{\beta}$-звездная по отношению $к$ точке $(0,0) u$

1) $(\beta+2) F(u)-\frac{\beta}{2} f(u)<0$, когда $u>0$;

2) $x_{1} \cdot \varphi^{\prime}\left(x_{1}\right) \geqslant(\beta+1) \varphi\left(x_{1}\right)$ в $\Omega$.

Тогда не существует нетривиальное положительное решение $и \in H^{2}(\Omega)$ для задачи (1), (2).

ПримеР 2. Рассмотрим случаи, когда $\varphi\left(x_{1}\right)=e^{-\left|x_{1}\right|^{-\delta}}(\delta>0)$ и $f(u)=\lambda u+|u|^{\gamma} u$, где $\lambda \leqslant 0, \gamma>0$. Покажем, что для любого $\gamma>0$ найдется $r(\gamma, \delta)$ такое, что задача $(1),(2)$ не имеет нетривиальньх решений $u \in H^{2}(\Omega)$, где $\Omega=B_{r(\gamma, \delta)}=\left\{\left(x_{1}, x_{2}\right)\right.$ | $\left.x_{1}^{2}+x_{2}^{2}<r^{2}(\gamma, \delta)\right\}$. Действительно, имеем $\varphi^{\prime}\left(x_{1}\right)=\operatorname{sign}\left(x_{1}\right)\left|x_{1}\right|^{-\delta-1} \delta e^{-\left|x_{1}\right|^{-\delta}}$. Следовательно,

$$
\begin{aligned}
x_{1} \varphi^{\prime}\left(x_{1}\right) \geqslant(\beta+1) \varphi\left(x_{1}\right) & \Longleftrightarrow \delta\left|x_{1}\right|^{-\delta} e^{-\left|x_{1}\right|^{-\delta}} \geqslant(\beta+1) e^{-\left|x_{1}\right|^{-\delta}} \\
& \Longleftrightarrow \delta\left|x_{1}\right|^{-\delta} \geqslant(\beta+1) \Longleftrightarrow\left|x_{1}\right| \leqslant\left(\frac{\delta}{\beta+1}\right)^{1 / \delta} .
\end{aligned}
$$

Если выберем $r(\gamma, \delta)=(\delta \gamma /(4+\gamma))^{1 / \delta}$, то $x_{1} \varphi^{\prime}\left(x_{1}\right) \geqslant((4+\gamma) / \gamma) \varphi\left(x_{1}\right)$ в $B_{r(\gamma, \delta)}$. Положив

$$
F(u)=\frac{\lambda u^{2}}{2}+\frac{|u|^{\gamma+2}}{\gamma+2}
$$

и $f(u)$ в условии 3$)$ теоремы 3 с $\beta=(4+\gamma) / \gamma$, получим

$$
\begin{aligned}
\int_{x_{1}^{2}+x_{2}^{2} \leqslant r^{2}(\gamma, \delta)}\left\{\left(\frac{4+2 \gamma}{\gamma}\right)\left(\frac{\lambda u^{2}}{2}+\frac{|u|^{\gamma+2}}{\gamma+2}\right)-\frac{2}{\gamma}\left(\lambda u^{2}+|u|^{\gamma+2}\right)\right\} d x_{1} d x_{2} \\
=\frac{1}{2} \int_{x_{1}^{2}+x_{2}^{2}=r^{2}(\gamma, \delta)}\left(\frac{\partial u}{\partial \nu}\right)^{2}\left(\nu_{1}^{2}+x_{1}^{8 / \gamma} \cdot \nu_{2}^{2}\right)\left\{x_{1} \cdot \nu_{1}+\left(\frac{4+\gamma}{\gamma}\right) x_{2} \cdot \nu_{2}\right\} d s \\
\quad+\int_{x_{1}^{2}+x_{2}^{2} \leqslant r^{2}(\gamma, \delta)}\left\{x_{1} \varphi^{\prime}\left(x_{1}\right) \varphi\left(x_{1}\right)-\left(\frac{4+\gamma}{\gamma}\right) \varphi^{2}\left(x_{1}\right)\right\}\left(\frac{\partial u}{\partial x_{2}}\right)^{2} d x_{1} d x_{2} .
\end{aligned}
$$

Taк как $x_{1} \varphi^{\prime}\left(x_{1}\right) \varphi\left(x_{1}\right) \geqslant((4+\gamma) / \gamma) \varphi^{2}\left(x_{1}\right)$ в $B_{r(\gamma, \delta)}$, тО

$$
\begin{aligned}
& \lambda \int_{x_{1}^{2}+x_{2}^{2} \leqslant r^{2}(\gamma, \delta)} u^{2} d x_{1} d x_{2} \\
& \quad \geqslant \frac{1}{2} \int_{x_{1}^{2}+x_{2}^{2}=r^{2}(\gamma, \delta)}\left(\frac{\partial u}{\partial \nu}\right)^{2}\left(x_{1}^{2}+x_{1}^{8 / \gamma} \cdot x_{2}^{2}\right)\left\{x_{1}^{2}+\left(\frac{4+\gamma}{\gamma}\right) x_{2}^{2}\right\} d s
\end{aligned}
$$

При $\lambda<0$ имеем

$$
\begin{aligned}
0 & >\lambda \int_{x_{1}^{2}+x_{2}^{2} \leqslant r^{2}(\gamma, \delta)} u^{2} d x_{1} d x_{2} \\
& \geqslant \frac{1}{2} \int_{x_{1}^{2}+x_{2}^{2}=r^{2}(\gamma, \delta)}\left(\frac{\partial u}{\partial \nu}\right)^{2}\left(x_{1}^{2}+x_{1}^{8 / \gamma} \cdot x_{2}^{2}\right)\left\{x_{1}^{2}+\left(\frac{4+\gamma}{\gamma}\right) x_{2}^{2}\right\} d s \geqslant 0
\end{aligned}
$$


которое ведет к противоречию. Если $\lambda=0$, выводим, что

$$
\frac{1}{2} \int_{x_{1}^{2}+x_{2}^{2}=r^{2}(\gamma, \delta)}\left(\frac{\partial u}{\partial \nu}\right)^{2}\left(x_{1}^{2}+x_{1}^{8 / \gamma} \cdot x_{2}^{2}\right)\left\{x_{1}^{2}+\left(\frac{4+\gamma}{\gamma}\right) x_{2}^{2}\right\} d s=0 .
$$

Следовательно,

$$
\left.\frac{\partial u}{\partial \nu}\right|_{x_{1}^{2}+x_{2}^{2}=r^{2}(\gamma, \delta)} \equiv 0
$$

Из теоремы о единственности следует, что $u \equiv 0$.

Теперь вернемся к случаю $\varphi^{2}\left(x_{1}\right)=x_{1}^{2 k}$.

3. Фундаментальное решение и преобразование Кельвина. Определим полярные координаты следуюшим образом:

$$
\begin{gathered}
x_{1}=\rho \operatorname{sign}(\sin \theta)|\sin \theta|^{1 /(k+1)}, \quad x_{2}=\frac{\rho^{k+1}}{k+1} \cos \theta, \\
d x_{1} d x_{2}=\frac{\rho^{k+1}}{k+1}|\sin \theta|^{-k /(k+1)} d \rho d \theta .
\end{gathered}
$$

Мы также используем следующие координаты:

$$
\begin{gathered}
\rho\left(x_{1}, x_{2}\right)=\left(x_{1}^{2 k+2}+(k+1)^{2} x_{2}^{2}\right)^{1 /(2 k+2)}, \\
\omega=|\sin \theta|^{1 /(k+1)} \operatorname{sign}(\sin \theta)=\frac{x_{1}}{\left(x_{1}^{2 k+2}+(k+1)^{2} x_{2}^{2}\right)^{1 /(2 k+2)}}, \\
G_{k}=\omega^{2 k}\left(\frac{\partial^{2}}{\partial \rho^{2}}+\frac{k+1}{\rho} \frac{\partial}{\partial \rho}\right)+\frac{1}{\rho^{2}}\left\{\left(1-\omega^{2 k+2}\right) \frac{\partial^{2}}{\partial \omega^{2}}-(k+1) \omega^{2 k+1} \frac{\partial}{\partial \omega}\right\} .
\end{gathered}
$$

Tеорема 6. Пусть $E_{k}\left(x_{1}, x_{2}\right)=\rho^{-k}$, тогда

$$
G_{k} E_{k}\left(x_{1}, x_{2}\right)=-\frac{2 k}{k+1} \sqrt{\pi} \frac{\Gamma((k+1) /(2 k))}{\Gamma((2 k+1) /(2 k))} \delta\left(x_{1}, x_{2}\right) .
$$

ДокАЗАтЕльство. Сначала покажем, что $E_{k}\left(x_{1}, x_{2}\right) \in L_{\mathrm{loc}}^{(k+2) / k-\tau}\left(\mathbb{R}^{2}\right)$ для любого достаточно малого положительного $\tau$. Так как $E_{k}\left(x_{1}, x_{2}\right) \in C^{\infty}\left(\mathbb{R}^{2} \backslash 0\right)$, достаточно доказать, что $E_{k}\left(x_{1}, x_{2}\right) \in L^{(k+2) / k-\tau}\left(B_{\rho}\right)$, где $B_{\rho}=\left\{\left(x_{1}, x_{2}\right) \mid \rho\left(x_{1}, x_{2}\right)<\rho\right\}$. Имеем

$$
\begin{aligned}
\int_{B_{\rho}}\left\{E_{k}\left(x_{1}, x_{2}\right)\right\}^{(k+2) / k-\tau} d x_{1} d x_{2} & =\int_{0}^{2 \pi} \frac{|\sin \theta|^{-k /(k+1)}}{k+1} d \theta \int_{0}^{\rho} s^{k+1}\left(s^{-k}\right)^{(k+2) / k-\tau} d s \\
& \leqslant C \int_{0}^{\rho} s^{-1+\tau k} d s<\infty .
\end{aligned}
$$

Заметим также, что $E_{k}\left(x_{1}, x_{2}\right) \notin L_{\text {loc }}^{(k+2) / k}\left(\mathbb{R}^{2}\right)$. Легко видеть, что $G_{k} E_{k}\left(x_{1}, x_{2}\right)=0$, если $\left(x_{1}, x_{2}\right) \neq(0,0)$. Обозначим $\Omega_{\varepsilon}=\left\{\left(x_{1}, x_{2}\right) \in \mathbb{R}^{2} \mid \rho\left(x_{1}, x_{2}\right) \geqslant \varepsilon\right\}$. В силу формулы Грина

$$
\begin{aligned}
& -\int_{\Omega_{\varepsilon}} E_{k}\left(x_{1}, x_{2}\right) G_{k} v\left(x_{1}, x_{2}\right) d x_{1} d x_{2}=\int_{\Omega_{\varepsilon}} G_{k} E_{k}\left(x_{1}, x_{2}\right) v\left(x_{1}, x_{2}\right) d x_{1} d x_{2} \\
& \quad+\int_{\rho=\varepsilon}\left\{v\left(x_{1}, x_{2}\right) \cdot \nu_{1} \cdot \frac{\partial E_{k}\left(x_{1}, x_{2}\right)}{\partial x_{1}}+v\left(x_{1}, x_{2}\right) \cdot \nu_{2} \cdot x_{1}^{2 k} \cdot \frac{\partial E_{k}\left(x_{1}, x_{2}\right)}{\partial x_{2}}\right\} d s \\
& \quad-\int_{\rho=\varepsilon}\left\{E_{k}\left(x_{1}, x_{2}\right) \cdot \nu_{1} \cdot \frac{\partial v\left(x_{1}, x_{2}\right)}{\partial x_{1}}+E_{k}\left(x_{1}, x_{2}\right) \cdot \nu_{2} \cdot x_{1}^{2 k} \cdot \frac{\partial v\left(x_{1}, x_{2}\right)}{\partial x_{2}}\right\} d s
\end{aligned}
$$


для любого $v \in C_{0}^{1}(\Omega)$.

Поскольку

$$
d s=\frac{1}{k+1}\left(\rho^{2}|\sin \theta|^{-2 k /(k+1)} \cos ^{2} \theta+\rho^{2 k+2} \sin ^{2} \theta\right)^{1 / 2}
$$

и

$$
\nu=\left(\nu_{1}, \nu_{2}\right)=\left(\frac{x_{1}^{2 k+1}}{\left(x_{1}^{4 k+2}+(k+1)^{2} x_{2}^{2}\right)^{1 / 2}}, \frac{(k+1) x_{2}}{\left(x_{1}^{4 k+2}+(k+1)^{2} x_{2}^{2}\right)^{1 / 2}}\right),
$$

имеем

$$
\begin{aligned}
\left\{\nu_{1}\right. & \left.\cdot \frac{\partial E_{k}\left(x_{1}, x_{2}\right)}{\partial x_{1}}+\nu_{2} \cdot x_{1}^{2 k} \cdot \frac{\partial E_{k}\left(x_{1}, x_{2}\right)}{\partial x_{2}}\right\}\left.\right|_{\rho=\varepsilon} \\
& =k x_{1}^{2 k} \varepsilon^{-k}\left(x_{1}^{4 k+2}+(k+1)^{2} x_{2}^{2}\right)^{-1 / 2} \\
& =-k \varepsilon^{k}|\sin \theta|^{2 k /(k+1)}\left(\varepsilon^{4 k+2}|\sin \theta|^{(4 k+2) /(k+1)}+\varepsilon^{2 k+2} \cos ^{2} \theta\right)^{-1 / 2} .
\end{aligned}
$$

Следовательно,

$$
\begin{aligned}
\int_{\rho=\varepsilon} & \left\{\nu_{1} \cdot \frac{\partial E_{k}\left(x_{1}, x_{2}\right)}{\partial x_{1}}+\nu_{2} \cdot x_{1}^{2 k} \cdot \frac{\partial E_{k}\left(x_{1}, x_{2}\right)}{\partial x_{2}}\right\} v\left(x_{1}, x_{2}\right) d s \\
= & \int_{0}^{2 \pi} v(\varepsilon, \theta)\left(-\frac{k}{k+1}\right) \varepsilon^{k}|\sin \theta|^{2 k /(k+1)} \\
& \times\left(\frac{\varepsilon^{2}|\sin \theta|^{-2 k /(k+1)} \cos ^{2} \theta+\varepsilon^{2 k+2} \sin ^{2} \theta}{\varepsilon^{4 k+2}|\sin \theta|^{(4 k+2) /(k+1)}+\varepsilon^{2 k+2} \cos ^{2} \theta}\right)^{1 / 2} d \theta \\
= & \int_{0}^{2 \pi} v(\varepsilon, \theta)\left(-\frac{k}{k+1}\right)|\sin \theta|^{k /(k+1)} d \theta .
\end{aligned}
$$

Устремляя $\varepsilon \rightarrow 0$, получим $v(\varepsilon, \theta)=V(\varepsilon, \theta)+v(0,0)$, где $V(\varepsilon, \theta) \rightarrow 0$ равномерно по $\theta$ при $\varepsilon \rightarrow 0$. Это означает, что

$$
\begin{gathered}
-\frac{k}{k+1} \int_{0}^{2 \pi} v(\varepsilon, \theta)|\sin \theta|^{k /(k+1)} d \theta \rightarrow-\frac{k}{k+1} v(0,0) \int_{0}^{2 \pi}|\sin \theta|^{k /(k+1)} d \theta \\
=-\frac{2 k}{k+1} \sqrt{\pi} \frac{\Gamma((k+1) /(2 k))}{\Gamma((2 k+1) /(2 k))} v(0,0) \quad \text { при } \varepsilon \rightarrow 0 .
\end{gathered}
$$

Оценим теперь выражение

$$
-\int_{\rho=\varepsilon}\left\{E_{k}\left(x_{1}, x_{2}\right) \cdot \nu_{1} \cdot \frac{\partial v\left(x_{1}, x_{2}\right)}{\partial x_{1}}+E_{k}\left(x_{1}, x_{2}\right) \cdot \nu_{2} \cdot x_{1}^{2 k} \cdot \frac{\partial v\left(x_{1}, x_{2}\right)}{\partial x_{2}}\right\} d s .
$$

\section{Имеем}

$$
\begin{aligned}
& \left|-\int_{\rho=\varepsilon}\left\{E_{k}\left(x_{1}, x_{2}\right) \cdot \nu_{1} \cdot \frac{\partial v\left(x_{1}, x_{2}\right)}{\partial x_{1}}+E_{k}\left(x_{1}, x_{2}\right) \cdot \nu_{2} \cdot x_{1}^{2 k} \cdot \frac{\partial v\left(x_{1}, x_{2}\right)}{\partial x_{2}}\right\} d s\right| \\
& \quad \leqslant C \int_{\rho=\varepsilon}\left|E_{k}\left(x_{1}, x_{2}\right)\right| \cdot\left(\left|\nu_{1}\right|+\left|\nu_{2} \cdot x_{1}^{2 k}\right|\right) d s=\int_{\rho=\varepsilon} \varepsilon^{-k} \frac{\left|x_{1}\right|^{2 k+1}+(k+1) x_{1}^{2 k}\left|x_{2}\right|}{\left(x_{1}^{4 k+2}+(k+1)^{2} x_{2}^{2}\right)^{1 / 2}} d s \\
& \quad=\frac{1}{k+1} \int_{0}^{2 \pi} \varepsilon^{k}|\sin \theta|^{2 k /(k+1)}\left(\varepsilon|\sin \theta|^{1 /(k+1)}+\varepsilon^{k+1}|\cos \theta|\right) \varepsilon^{-k}|\sin \theta|^{-k /(k+1)} d \theta \\
& \quad=\frac{1}{k+1} \int_{0}^{2 \pi}|\sin \theta|^{k /(k+1)}\left(\varepsilon|\sin \theta|^{1 /(k+1)}+\varepsilon^{k+1}|\cos \theta|\right) d \theta \rightarrow 0 \quad \text { при } \varepsilon \rightarrow 0 .
\end{aligned}
$$


В силу (3)-(5) заключаем, что

$$
\begin{aligned}
\left(G_{k} E_{k}\left(x_{1}, x_{2}\right), v\left(x_{1}, x_{2}\right)\right) & =\left(E_{k}\left(x_{1}, x_{2}\right), G_{k} v\left(x_{1}, x_{2}\right)\right) \\
& =\lim _{\varepsilon \rightarrow 0} \int_{\rho \geqslant \varepsilon} E_{k}\left(x_{1}, x_{2}\right) G_{k} v\left(x_{1}, x_{2}\right) \\
& =-\frac{2 k}{k+1} \sqrt{\pi} \frac{\Gamma((k+1) /(2 k))}{\Gamma((2 k+1) /(2 k))} v(0,0) .
\end{aligned}
$$

ЗАмЕЧАнИЕ 1 . Поскольку $G_{k}$ инвариантно относительно преобразования $\left(x_{1}, x_{2}\right) \rightarrow$ $\left(x_{1}, x_{2}+c\right)$, мы также имеем

$$
G_{k} E_{k}\left(x_{1}, x_{2}+c\right)=-\frac{2 k}{k+1} \sqrt{\pi} \frac{\Gamma((k+1) /(2 k))}{\Gamma((2 k+1) /(2 k))} \delta(0,-c) .
$$

ЗАмЕчАниЕ 2 . Если $x_{1} \neq 0$, ядро ведет себя как $\log$, однако оно имеет скачок при приближении к отрезку $x_{1}=0$.

Введем преобразование Кельвина

$$
x_{1}=\frac{y_{1}}{\left\{y_{1}^{2 k+2}+(k+1)^{2} y_{2}^{2}\right\}^{1 /(k+1)}}, \quad x_{2}=\frac{y_{2}}{\left\{y_{1}^{2 k+2}+(k+1)^{2} y_{2}^{2}\right\}} .
$$

Легко видеть, что

$$
y_{1}=\frac{x_{1}}{\left\{x_{1}^{2 k+2}+(k+1)^{2} x_{2}^{2}\right\}^{1 /(k+1)}}, \quad y_{2}=\frac{x_{2}}{\left\{x_{1}^{2 k+2}+(k+1)^{2} x_{2}^{2}\right\}}
$$

и

$$
\left\{x_{1}^{2 k+2}+(k+1)^{2} x_{2}^{2}\right\}=\frac{1}{\left\{y_{1}^{2 k+2}+(k+1)^{2} y_{2}^{2}\right\}} .
$$

При преобразовании Кельвина кривая $\left\{y_{1}^{2 k+2}+(k+1)^{2}\left(y_{2}-1\right)^{2}\right\} \leqslant(k+1)^{2}$ переходит в $x_{2} \geqslant 1 /(2 k+2)$. Действительно,

$$
\begin{aligned}
x_{2} \geqslant \frac{1}{2 k+2} & \Longleftrightarrow \frac{y_{2}}{\left\{y_{1}^{2 k+2}+(k+1)^{2} y_{2}^{2}\right\}} \geqslant \frac{1}{2 k+2} \\
& \Longleftrightarrow\left\{y_{1}^{2 k+2}+(k+1)^{2} y_{2}^{2}\right\} \leqslant 2(k+1) y_{2} .
\end{aligned}
$$

Это означает, что $\left\{y_{1}^{2 k+2}+(k+1)^{2}\left(y_{2}-1\right)^{2}\right\} \leqslant(k+1)^{2}$.

Заметим, что

$$
\begin{aligned}
G_{k}(u \cdot v) & =\frac{\partial^{2}}{\partial x_{1}^{2}}(u v)+x_{1}^{2 k} \frac{\partial^{2}}{\partial x_{2}^{2}}(u v) \\
& =v \frac{\partial^{2} u}{\partial x_{1}^{2}}+u \frac{\partial^{2} v}{\partial x_{1}^{2}}+2 \frac{\partial u}{\partial x_{1}} \frac{\partial v}{\partial x_{1}}+x_{1}^{2 k}\left\{v \frac{\partial^{2} u}{\partial x_{2}^{2}}+u \frac{\partial^{2} v}{\partial x_{2}^{2}}+2 \frac{\partial u}{\partial x_{2}} \frac{\partial v}{\partial x_{2}}\right\} \\
& =v G_{k} u+u G_{k} v+2\left\{\frac{\partial u}{\partial x_{1}} \frac{\partial v}{\partial x_{1}}+x_{1}^{2 k} \frac{\partial u}{\partial x_{2}} \frac{\partial v}{\partial x_{2}}\right\} .
\end{aligned}
$$

Пусть $v(y)=\rho^{k}(x(y)) u(x(y))$ и через $G_{k}^{(y)}, G_{k}^{(x)}$ обозначим оператор Грушина, действуюший по переменным $y$ и $x$. Подробное вычисление показывает (см. [6]), что

$$
G_{k}^{(y)} v(y)=\rho^{k+4}(x) G_{k}^{(x)} u(x) .
$$


4. Задача на собственное значение. Рассмотрим задачу

$$
G_{k} u+\lambda u+f(x)=0,
$$

где $\lambda \in \mathbb{C}, f \in L^{2}(\Omega)$ и $u \in S_{0, k}^{1,2}(\Omega)$. Сформулируем классические теоремы Гильберта-Шмидта, Ентча и $\Phi$ редгольма для $G_{k}$.

Теорема 7. 1) Множество собственных значений $\left\{\lambda_{j}\right\}$ непусто, расположено на вещественной оси $\mathbb{R}^{+}=\{x \in \mathbb{R} \mid x>0\}$, не более чем счетно и не имеет конечных предельных точек. Каждое характеристическое число имеет конечную кратность, система собственных функиий $\left\{\psi_{j}\right\}$ мохет быть выбрана ортонормированной в $L^{2}(\Omega)$, и она ортогональна и полна в $L^{2}(\Omega)$ u $S_{0, k}^{1,2}(\Omega)$.

2) $\psi_{1}\left(x_{1}, x_{2}\right)$ не меняет знак в $\Omega$ (напомним, что $\Omega$ связно) $и \lambda_{1}$ положительно u просто.

3) Если $\lambda \neq \lambda_{j}, j=1,2, \ldots$, то уравнение (6) однозначно разрешимо при любом свободном члене $f \in L^{2}(\Omega)$. Если $\lambda=\lambda_{j}$, то для разрешимости (6) необходимо $u$ достаточно, чтобы

$$
\left(f, \psi_{j+i}\right)_{L^{2}(\Omega)}=0, \quad i=0, \ldots, r_{j}-1,
$$

где $\psi_{j}, \psi_{j+1}, \ldots, \psi_{j+r_{j}-1}$ - собственные функиии, соответствующие собственно-

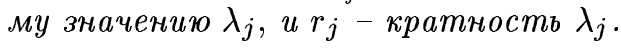

ДокАЗАТЕЛЬСТво. Опускаем детали.

Теперь нас интересует гладкость собственных функций. Из гипоэллиптичности $G_{k}$ выводим, что $u \in C^{\infty}(\Omega)$. Во всех точках $\left(x_{1}, x_{2}\right) \in \partial \Omega, x_{1} \neq 0$, выполнено $u \in$ $C^{\infty}\left(O\left(x_{1}, x_{2}\right) \cap \Omega\right)$, так как $G_{k}$ эллиптичен в них. Здесь через $O\left(x_{1}, x_{2}\right)$ обозначена некоторая окрестность $\left(x_{1}, x_{2}\right)$. Более того, если $\left(0, x_{2}\right) \in \partial \Omega$ и $\nu\left(0, x_{2}\right) \nsim(0,1)$, то из результата Кона-Ниренберга [7] вытекает, что $u \in C^{\infty}\left(O\left(x_{1}, x_{2}\right) \cap \Omega\right)$ (по крайней мере для $k=1)$. Мы покажем некоторые результаты о гладкости собственных функций для $\left(0, x_{2}\right)$ и $\nu\left(0, x_{2}\right) \sim(0,1)$.

Tеорема 8. А) Предположим, что

1) $G_{k} u+\lambda a\left(x_{1}, x_{2}\right) u=0$ в $\Omega$, где $a\left(x_{1}, x_{2}\right) \in C^{\infty}(\bar{\Omega}) u u \in S_{0, k}^{1,2}(\Omega)$;

$2)$ если $\left(0, \widetilde{x}_{2}\right) \in \partial \Omega$, то существует некоторая окрестность $O\left(0, \widetilde{x}_{2}\right)$ такая, что $O\left(0, \widetilde{x}_{2}\right) \cap \partial \Omega=$ линия $\left(x_{1}, \widetilde{x}_{2}\right)$, где $-c_{1}<x_{1}<c_{1}$.

Tогда $u \in C^{\infty}\left(\overline{O\left(0, \widetilde{x}_{2}\right) \cap \Omega}\right)$.

Б) Предположим, что

1) $G_{k} u+\lambda u=0$ в $\Omega$, где $a\left(x_{1}, x_{2}\right) \in C^{\infty}(\bar{\Omega}) u u \in S_{0, k}^{1,2}(\Omega)$;

2) если $\left(0, \widetilde{x}_{2}\right) \in \partial \Omega$, то существует некоторая окрестность $O\left(0, \widetilde{x}_{2}\right)$ такая, что $O\left(0, \widetilde{x}_{2}\right) \cap \partial \Omega=\left\{(k+1)^{2}\left(x_{2}-\widetilde{\widetilde{x}}_{2}\right)^{2}+x_{1}^{2 k+2}=(k+1)^{2}\right\} \cap \partial \Omega$.

Тогда $u \in C^{\infty}\left(\overline{O\left(0, \widetilde{x}_{2}\right) \cap \Omega}\right)$.

ДокАЗАТЕЛЬСтво. А) Поскольку $G_{k}$ инвариантно относительно преобразования $\left(x_{1}, x_{2}\right) \rightarrow\left(x_{1}, x_{2}+c\right)$, можно считать, что $\left(0, \widetilde{x}_{2}\right)=(0,0)$ и $\Omega \subset\left\{\left(x_{1}, x_{2}\right) \mid x_{2}>0\right\}$. Продолжим $a\left(x_{1}, x_{2}\right), u\left(x_{1}, x_{2}\right)$ из $\Omega$ в $\bar{\Omega}=\Omega \cap \Omega_{1}$, где $\Omega_{1}=\left\{\left(x_{1}, x_{2}\right) \mid\left(x_{1},-x_{2}\right) \in \Omega\right\}$ определяется как

$$
\widetilde{a}\left(x_{1}, x_{2}\right)= \begin{cases}a\left(x_{1}, x_{2}\right), & \text { если }\left(x_{1}, x_{2}\right) \in \widetilde{\Omega}, \\ a\left(x_{1},-x_{2}\right), & \text { если }\left(x_{1}, x_{2}\right) \in \Omega_{1},\end{cases}
$$




$$
\widetilde{u}\left(x_{1}, x_{2}\right)=\left\{\begin{array}{cl}
u\left(x_{1}, x_{2}\right), & \text { если }\left(x_{1}, x_{2}\right) \in \Omega, \\
-u\left(x_{1},-x_{2}\right), & \text { если }\left(x_{1}, x_{2}\right) \in \Omega_{1} .
\end{array}\right.
$$

Тогда $\widetilde{u}\left(x_{1}, x_{2}\right) \in C^{\infty}(O(0,0) \backslash(0,0)), a\left(x_{1}, x_{2}\right) \in C(O(0,0))$ и

$$
G_{k} \widetilde{u}+\lambda \widetilde{a}\left(x_{1}, x_{2}\right) \widetilde{u}=0 \quad \text { в } O(0,0) \backslash(0,0) .
$$

Из теоремы Шварца вытекает, что

$$
G_{k} \tilde{u}+\lambda \widetilde{a}\left(x_{1}, x_{2}\right) \widetilde{u}=\sum_{|\alpha| \leqslant m} C_{\alpha} \delta^{\alpha}(0,0)
$$

Заметим, что $\widetilde{u} \in L^{2}(O(0,0))$. Отсюда вытекает, что

$$
G_{k} \widetilde{u}+\lambda \widetilde{a}\left(x_{1}, x_{2}\right) \widetilde{u} \in H_{\mathrm{loc}}^{-2}(O(0,0)) .
$$

С другой стороны, $\delta^{\alpha} \notin H_{\mathrm{loc}}^{-2}(O(0,0))$, если $|\alpha| \geqslant 1$. Следовательно,

$$
G_{k} \widetilde{u}+\lambda \widetilde{a}\left(x_{1}, x_{2}\right) \widetilde{u}=C \delta(0,0)=C G_{k} E_{k}\left(x_{1}, x_{2}\right) .
$$

Это означает, что $G_{k}\left(\widetilde{u}-C E_{k}\right)=-\lambda \widetilde{a} \widetilde{u}$. Мы также имеем $\lambda \widetilde{a}\left(x_{1}, x_{2}\right) \widetilde{u} \in L^{2}(O(0,0))$, поэтому в силу теоремы Хёрмандера [8] выводим, что $\widetilde{u}-C E_{k} \in L_{\text {loc }}^{2}(O(0,0))$. Следовательно, $E_{k} \in L_{\text {loc }}^{2}(O(0,0))$, что приводит к противоречию, если $k \geqslant 2$, так как $E_{k} \notin$ $L_{\text {loc }}^{(k+2) / k}(\Omega)$. Рассмотрим случай $k=1$. В силу теоремы 2 имеем $\tilde{u} \in L_{\text {loc }}^{5}(O(0,0))$. Из теоремы Ротшильда-Стейна [9] вьводим, что $\widetilde{u}-C E_{k} \in L_{\text {loc }}^{5}(O(0,0))$. Следовательно, $E_{k} \in L_{\text {loc }}^{5}(O(0,0))$, что также ведет к противоречию, так как $E_{k} \notin L_{\text {loc }}^{3} O(0,0)$.

Б) Как и ранее, поскольку $G_{k}$ инвариантно относительно преобразования $\left(x_{1}, x_{2}\right) \rightarrow$ $\left(x_{1}, x_{2}+c\right)$, можно считать, что $\left(0, \widetilde{x}_{2}\right)=(0,0)$ и

$$
O(0,1) \cap \partial \Omega=\left\{(k+1)^{2}\left(x_{2}-1\right)^{2}+x_{1}^{2 k+2}=(k+1)^{2}\right\} \cap \partial \Omega .
$$

Пусть $v(y)=\rho^{k}(x) u(x)$, где $y_{1}, y_{2}$, как в преобразовании Кельвина. Тогда получим уравнение для $v(y)$

$$
\rho^{k+4}(y) G_{k}^{(y)} v(y)+\lambda \rho^{k}(y) v(y)=0 .
$$

Отсюда вытекает, что $G_{k} v(y)+\lambda \rho^{-4} v(y)=0$ в некоторой окрестности $O(0,1 /(2 k+2)) \cap$ $\left\{\left(y_{1}, y_{2}\right) \mid y_{2} \geqslant 1 /(2 k+2)\right\}$. Из части А) заключаем, что

$$
v(y) \in C^{\infty}\left(\overline{O\left(0, \frac{1}{2 k+2}\right) \cap\left\{\left(y_{1}, y_{2}\right) \mid y_{2}>\frac{1}{2 k+2}\right\}}\right) .
$$

Следовательно, $u\left(x_{1}, x_{2}\right)=v(y) \rho^{-k}\left(x_{1}, x_{2}\right) \in C^{\infty}(\overline{O(0,1) \cap \Omega})$. 
ПРимеР 3. Здесь приведем некоторое явное вычисление для собственных значений в квадрате. Пусть $\Omega=(-\pi / 2, \pi / 2) \times(-\pi / 2, \pi / 2)$. Будем искать собственные функции в виде $u\left(x_{1}, x_{2}\right)=f\left(x_{1}\right) \cdot g\left(x_{2}\right)$ с $f(-\pi / 2)=f(\pi / 2)=0$ и $g(-\pi / 2)=g(\pi / 2)=0$. Имеем

$$
f^{\prime \prime}\left(x_{1}\right) g\left(x_{2}\right)+x_{1}^{2 k} f\left(x_{1}\right) g^{\prime \prime}\left(x_{2}\right)+\lambda f\left(x_{1}\right) g\left(x_{2}\right)=0
$$

или

$$
g\left(x_{2}\right)\left\{f^{\prime \prime}\left(x_{1}\right)+\lambda f\left(x_{1}\right)\right\}=-x_{1}^{2 k} f\left(x_{1}\right) g^{\prime \prime}\left(x_{2}\right) .
$$

Следовательно,

$$
\frac{f^{\prime \prime}\left(x_{1}\right)+\lambda f\left(x_{1}\right)}{x_{1}^{2 k} f\left(x_{1}\right)}=-\frac{g^{\prime \prime}\left(x_{2}\right)}{g\left(x_{2}\right)}=C .
$$

Таким образом, приходим к задачам на собственные значения для $f\left(x_{1}\right), g\left(x_{2}\right)$

$$
\begin{gathered}
g^{\prime \prime}\left(x_{2}\right)+C g\left(x_{2}\right)=0, \quad g\left(-\frac{\pi}{2}\right)=g\left(\frac{\pi}{2}\right)=0, \\
f^{\prime \prime}\left(x_{1}\right)-C x_{1}^{2 k} f\left(x_{1}\right)+\lambda f\left(x_{1}\right)=0, \quad f\left(-\frac{\pi}{2}\right)=f\left(\frac{\pi}{2}\right)=0 .
\end{gathered}
$$

Из (7) заключаем, что $C=n^{2}$ и $g\left(x_{2}\right)=C \sin \left(x_{2}-\pi / 2\right) n$. Для каждого $C=n^{2}$ задача (8) имеет собственные значения $\lambda_{n}^{j}$ с условием $\lim _{j \rightarrow \infty} \lambda_{n}^{j}=\infty$, и каждое собственное значение имеет конечную кратность. Из свойства собственных значений для оператора Грушина также следует тот факт, что собственные значения для задачи

$$
f^{\prime \prime}\left(x_{1}\right)-n^{2} x_{1}^{2 k} f\left(x_{1}\right)+\lambda f\left(x_{1}\right)=0
$$

никогда не совпадают бесконечное число раз. Легко видеть, что система $\sin \left(x_{2}-\pi / 2\right) n \times$ $f_{n}^{j}\left(x_{1}\right)(n=1,2, \ldots \infty, j=1,2, \ldots \infty)$ полна. Следовательно, всеми собственными значениями для $G_{k}$ в квадрате служат только $\lambda_{n}^{j}$.

Автор выражает искреннюю благодарность Международному центру по теоретической физике за поддержку и гостеприимство.

\section{СПИСОК ЦИТИРОВАННОЙ ЛИТЕРАТУРЫ}

[1] Федий В. С. Об одном условии гипоэллиптичности // Матем. сб. 1971. Т. 85. С. 18-48.

[2] Jerison D. V. The Dirichlet problem for the Kohn Laplacian on the Heisenberg group. II // J. Funct. Anal. 1981. V. 43. P. 224-257.

[3] Tri N. M. Sobolev Imbedding Theorems for Degenerate Metric. Preprint ICTP. Teaneck, NJ: World Sci. Publ., 1995.

[4] Garofalo N., Lanconelli E. Existence and nonexistence results for semilinear equations on the Heisenberg group // Indiana Univ. Math. J. 1992. V. 41. P. 71-98.

[5] Похожаев С. И. О собственньх функциях уравнения $\Delta u+\lambda f(u)=0 / /$ Докл. АН СССР. 1965. T. 165. № 1. C. 36-39.

[6] Tri N. M. Superlinear Equations for Degenerate Elliptic Operators. Preprint ICTP. Teaneck, NJ: World Sci. Publ., 1995.

[7] Kohn J. J., Nirenberg L. Non-coercive boundary value problems // Comm. Pure Appl. Math. 1965. V. 18. P. 443-492.

[8] Hörmander L. Hypoelliptic second-order differential equations // Acta Math. 1967. V. 119. P. 147-171.

[9] Rothschild L. P., Stein E. M. Hypoelliptic differential operators and nilpotent groups // Acta Math. 1976. V. 137. P. 247-320.

Institute of Mathematics (Hanoi, Vietnam)

International Centre for Theoretical Phisics (Trieste, Italy)

Поступило

E-mail: triminh@ictp.trieste.it 